\title{
MEASURABLE SELECTIONS IN NORMED SPACES
}

\author{
by S. J. LEESE \\ (Received 12th June 1973)
}

It is sometimes desirable to know in what circumstances a measurable setvalued function admits a measurable selector; this problem occurs regularly in the theory of optimal control (see for example (3) and (7)). In this paper we demonstrate the existence of measurable selectors in two particular cases where the choice of selector has a simple geometrical interpretation, namely that of being a " nearest-point " selector, as is explained in detail below. This work derives in part from that of $\mathrm{C}$. Castaing, particularly from Théorème 3.4 of (2), of which this is an extension.

Following (1), (2), (6) and (8), we define a function $\Gamma$ from a measurable space $S$ (that is, a set on which is defined a $\sigma$-algebra of subsets called " measurable ") into the non-empty subsets of a topological space $X$ to be measurable if and only if for each closed set $F$ in $X$ the set

$$
\Gamma^{-}(F)=\{t \in S: \Gamma(t) \cap F \neq \phi\}
$$

is measurable. $\Gamma$ is described as a "measurable multifunction from $S$ into $X$ ". A function $f$ from $S$ into $X$ is called a selector for $\Gamma$ if $f(t) \in \Gamma(t)$ for all $t$ in $S ; f$ is measurable if and only if $f^{-1}(F)$ is measurable for every closed subset $F$ of $X$.

If $\Gamma$ is a measurable multifunction from $S$ into $X$, and if $B$ is any closed set in $X$, then we define the refinement of $\Gamma$ by the set $B$ to be the multifunction $\Gamma_{B}$, where

and

$$
\Gamma_{B}(t)=\Gamma(t) \cap B \text { for } t \in \Gamma^{-}(B)
$$

$$
\Gamma_{B}(t)=\Gamma(t) \quad \text { otherwise. }
$$

$\Gamma_{B}$ is measurable, since if $C$ is a closed subset of $X$,

$$
\Gamma_{B}^{-}(C)=\left(\Gamma^{-}(B \cap C)\right) \cup\left(\Gamma^{-}(C) \backslash \Gamma^{-}(B)\right)
$$

which is clearly measurable (see also (8)).

Consider now a descending sequence $\left(\Gamma_{n}\right)$ of closed-valued measurable multifunctions from $S$ into $X$. Then in order to show that the multifunction $\Gamma=\bigcap \Gamma_{n}$, defined by

$$
\Gamma(t)=\bigcap_{n=1}^{\infty} \Gamma_{n}(t) \text { for each } t \text { in } S,
$$

is also measurable, it is sufficient to show that we have

$$
\Gamma^{-}(A)=\bigcap_{n=1}^{\infty} \Gamma_{n}^{-}(A)
$$


for each closed set $A$ in $X$. Conditions which ensure this, and also that $\Gamma(t)$ be non-empty for each $t$, are

(i) for each $t, \Gamma_{n}(t)$ is compact for some $n$

or (ii) $X$ is a complete metric space, and for each $t$ the diameter of the set $\Gamma_{n}(t)$ converges to zero as $n \rightarrow \infty$.

If condition (ii) holds, then $\Gamma(t)$ contains but a single point $\gamma(t) ; \gamma$ is therefore a measurable selector for $\Gamma_{1}$. We shall make use of these facts in what follows.

Lemma. Let $S$ be a measurable space and $(X, d)$ a metric space. Let $\Gamma: S \rightarrow X$ be a measurable multifunction with values compact and non-empty, and let $x_{0}$ be $a($ fixed) point of $X$. For each $t$, let

$$
\Delta(t)=\left\{x \in \Gamma(t): d\left(x_{0}, x\right)=d\left(x_{0}, \Gamma(t)\right)\right\}
$$

the set of all points of $\Gamma(t)$ at shortest distance from $x_{0}$. Then $\Delta$ is also a measurable multifunction.

Proof. We note first that the function $x \rightarrow d\left(x, x_{0}\right)$ is continuous and so attains its minimum on $\Gamma(t)$. Hence, for all $t, \Delta(t)$ is compact and non-empty.

Arrange the non-negative rationals in a sequence $\left(r_{n}\right)$ and let

$$
B_{n}=\left\{x \in X: d\left(x, x_{0}\right) \leqq r_{n}\right\} .
$$

Write $\Gamma_{0}=\Gamma$ and define $\Gamma_{n}$ recursively so that $\Gamma_{n}$ is the refinement of $\Gamma_{n-1}$ by the closed set $B_{n}$. Then the sequence $\left(\Gamma_{n}\right)$ satisfies condition (i) mentioned above, and so $\bigcap \Gamma_{n}$ is a measurable multifunction. The proof of the lemma will be complete when we have shown that

for all $t$.

$$
\Delta(t)=\bigcap_{n=1}^{\infty} \Gamma_{n}(t)
$$

To see this, if $x \in \Delta(t)$, then $d\left(x, x_{0}\right)=d\left(x_{0}, \Gamma(t)\right)$, It follows by induction on $n$ that $x \in \Gamma_{n}(t)$ for all $n$. Conversely, suppose that $x \in \Gamma_{n}(t)$ for all $n$. Then $r_{m} \geqq d\left(x, x_{0}\right) \geqq d\left(x_{0}, \Gamma(t)\right)$ for every rational $r_{m}$ greater than $d\left(x_{0}, \Gamma(t)\right)$. (For if $r_{m} \geqq d\left(x_{0}, \Gamma(t)\right)$, then $B_{m}$ meets $\Gamma(t)$. Hence $\Gamma_{p}(t) \subseteq B_{m}$ for $p \geqq m$, and so $x \in B_{m}$.) Therefore $d\left(x, x_{0}\right)=d\left(x_{0}, \Gamma(t)\right)$ and so $x \in \Delta(t)$. This proves (iii) and completes the proof of the Lemma.

The problem of finding a selector in a metric (or metrisable) space is now reduced to that of finding a selector for $\Delta$. If $\Delta$ contains a single point $\delta(t)$ for all $t$, then we need look no further. This happens in a wide class of normed spaces when $\Gamma(t)$ is assumed to be convex.

A normed linear space $E$ is said to be rotund (or strictly convex) if for any two distinct elements $x, y$ in $E$ such that $\|x\|=1$ and $\|y\|=1$, then $\|x+y\|<2$.

A normed linear space $E$ is said to be strictly normable if it has an equivalent strictly convex norm (for a discussion of strict convexity, see (4) p. 342 et seq.). 


\section{MEASURABLE SELECTIONS IN NORMED SPACES}

In particular it is known that every reflexive Banach space is strictly normable; this is proved in (5).

Theorem 1. Let $S$ be a measurable space, and $E$ a strictly normable space. Then every measurable multifunction $\Gamma$ from $S$ into $E$ with values which are non-empty, convex and compact has a measurable selector.

Proof. Using the notation of the Lemma, let $x_{0}=0$, the zero element; we may assume that the norm on $E$ is strictly convex. Then

$$
\Delta(t)=\{u \in \Gamma(t):\|u\|=d(0, \Gamma(t))\}
$$

and $\Delta$ is measurable, by the Lemma; moreover $\Delta(t)$ is compact, convex and non-empty for each $t$. Also $\Delta(t)$ contains just one point $\delta(t)$; for if $x \in \Delta(t)$ and $y \in \Delta(t)$, then $\frac{1}{2}(x+y) \in \Delta(t)$. Since the norm is strictly convex, $x \neq y$ would imply $\left\|\frac{1}{2}(x+y)\right\|<\|x\|$ strictly, which contradicts the definition of $\Delta$.

Thus $\delta$ is the required measurable selector.

A normed linear space is said to be uniformly convex if it always follows from

$$
\left\|x_{n}\right\| \leqq 1,\left\|y_{n}\right\| \leqq 1 \text { and } \lim _{n \rightarrow \infty}\left\|x_{n}+y_{n}\right\|=2 \text { that } \lim _{n \rightarrow \infty}\left\|x_{n}-y_{n}\right\|=0 \text {. }
$$

A normed linear space is said to be uniformly normable if it has an equivalent uniformly convex norm. Uniform convexity is discussed in (4), Section 26. In particular the standard Banach spaces $l^{p}$ and $L^{p}$ are uniformly convex for $1<p<\infty$. We note the following: if $\left(A_{n}\right)$ is a sequence of convex sets in a uniformly convex space and $\left(\beta_{n}\right)$ a sequence of non-negative real numbers, with limit $\alpha$, such that for each $n$

$$
\alpha \leqq\|x\| \leqq \beta_{n} \text { for all } x \in A_{n},
$$

then the diameter of $A_{n}$ tends to zero as $n \rightarrow \infty$.

Proof. This is obvious if $\alpha=0$; otherwise if $x_{n}, y_{n} \in B_{n}$ where $\beta_{n} B_{n}=A_{n}$, then $\left\|x_{n}\right\| \leqq 1,\left\|y_{n}\right\| \leqq 1$ and

$$
\left\|\frac{1}{2}\left(x_{n}+y_{n}\right)\right\| \geqq \frac{\alpha}{\beta_{n}} \rightarrow 1 \text {. }
$$

Therefore $\left\|x_{n}-y_{n}\right\| \rightarrow 0$, so $\operatorname{diam}\left(B_{n}\right) \rightarrow 0$, and so $\operatorname{diam}\left(A_{n}\right) \rightarrow 0$.

Theorem 2. Let $S$ be a measurable space, and $E$ a uniformly normable Banach space. Then every measurable multifunction from $S$ into $E$ with values closed, convex and non-empty has a measurable selector.

Proof. We consider $E$ with a uniformly convex norm; $E$ is a Banach space with respect to this norm. We proceed as in the proof of the Lemma. The proof will be complete when we have shown that the sequence $\left(\Gamma_{n}\right)$ obtained by this method satisfies the second of the two conditions discussed in the introduction. That is, it is sufficient to prove that for each $t$ in $S$

$$
\operatorname{diam}\left(\Gamma_{n}(t)\right) \rightarrow 0 \text { as } n \rightarrow \infty \text {. }
$$


This follows from the preceding remark: take $A_{n}=\Gamma_{n}(t), \alpha=d(0, \Gamma(t))$ and define $\left(\beta_{n}\right)$ recursively as follows:

and

$$
\beta_{n}=\min \left(\beta_{n-1}, r_{n}\right) \text { if } \alpha \leqq r_{n},
$$

$$
\beta_{n}=\beta_{n-1} \quad \text { otherwise, for } n>1 \text {. }
$$

We take $\beta_{1}$ to be equal to $r_{k}$, where $k$ is the first integer such that $r_{k} \geqq \alpha$. Then $\beta_{n}$ satisfies the required condition for $n \geqq k$.

Hence $\Delta(t)$ contains just one point $\gamma(t)$, and $\gamma$ is the required selector.

It is possible to relax the conditions on $E$ in the " nearest-point " problem at the expense of placing greater restrictions on $S$. In (1), the authors have relaxed the uniform convexity condition, by restricting $S$ to be a locally compact Hausdorff space in which every compact subset is metrisable and also by modifying the definition of measurability.

\section{Acknowledgements}

I am grateful to the Science Research Council, who have provided the financial support necessary for this work, and also to Professor A. P. Robertson for his helpful criticisms and suggestions.

\section{REFERENCES}

(1) C. J. Himmelberg, M. Q. Jacobs and F. S. Van Vleck, Measurable multifunctions, selectors, and Filippov's implicit functions lemma, J. Math. Anal. Appl. 25 (1969), 276-284.

(2) C. CAstaing, Sur les multiapplications mesurables, Rev. Française Informat. Recherche Opérationelle 1 (1967), 91-126.

(3) A. F. FILIPPov, On certain questions in the theory of optimal control, SIAM J. Control 1 (1962), 76-84.

(4) G. Köthe (tr. D. J. H. GaRunG), Topological Vector Spaces I (SpringerVerlag, Berlin, 1969).

(5) J. Lindenstrauss, On nonseparable reflexive Banach spaces, Bull. Amer. Math. Soc. 72 (1966), 967-970.

(6) R. T. RockafellaR, Measurable dependence on convex sets and functions on parameters, J. Math. Anal. Appl. 28 (1969), 4-25.

(7) E. J. MCShane and R. B. WARFELD Jr., On Filippov's implicit functions lemma, Proc. Amer. Math. Soc. 18 (1967), 41-47.

(8) A. P. Robertson, On measurable selections, Proc. Roy. Soc. Edinburgh (Sect. A) 71 (1973).

Department of Mathematics

UNIVERSITY OF WeSTERN AUSTRALIA

NEDLANDS

Western Australia 6009 